\title{
Wavelet Domain Filtering for Nuclear Medicine Imaging
}

\author{
Robert D. Nowak, ${ }^{1}$, David J. Nowak, ${ }^{2}$ Richard G. Baraniuk, ${ }^{3}$ and Robert S. Hellman ${ }^{4}$ \\ ${ }^{1}$ Michigan State University, East Lansing, MI, 48824-1226 \\ Email: rnowak@egr.msu.edu (corresponding author) \\ ${ }^{2}$ Independent Consultant, Milwaukee, WI \\ ${ }^{3}$ Rice University, Houston, TX \\ ${ }^{4}$ Medical College of Wisconsin, Milwaukee, WI
}

\begin{abstract}
We propose a new filtering/estimation method for nuclear medicine imaging. The statistical method of crossvalidation is used to design optimal wavelet domain filters for improved image estimation. The quality of the resulting images is much better than standard image estimates, in both visual and mean square error senses. Moreover, experiments have shown that, using the new estimate, we can reduce the acquisition time by a factor of two and still retain high image quality.
\end{abstract}

\section{INTRODUCTION}

This paper reports on the development of a new waveletbased filtering algorithm for nuclear medicine imaging. Wavelet-based filtering is a relatively recent advance in digital signal processing (DSP) [1]. Wavelet-based filtering offers significant theoretical advantages over classical linear filters $[1,4]$, and real-world applications have demonstrated the outstanding performance of these new methods [3].

The basic DSP problem in nuclear medicine imaging is to design a digital filter that that recovers the signal (intensity image) from the noisy observation (observed counts). Nuclear medicine images have a low signal-to-noise ratio (SNR) compared to other imaging modalities. There are several sources of noise in nuclear medicine: low count levels, scatter, attenuation, and electronic noises in the detector/camera. These noises can severely degrade image quality.

The standard imaging technique forms an image based directly on the total counts detected over a fixed observation period. This image is an estimate of the underlying intensity of the decay process, and is proportional to the Maximum Likelihood Estimate (MLE) (assuming a Poisson model and ignoring noises other than those due to low counts). The MLE has the desirable property that it is unbiased. However, because of the noise in the imaging process, the MLE image appears noisy and has a large variance about its true mean (the underlying intensity of the Poisson process). A simple approach to noise reduction is linear filtering or smoothing of the MLE. However, linear filtering of the MLE reduces the noise at the expense of blurring important details in the signal of interest (e.g., edges in the image).

In this paper, we introduce a new estimation/filtering method for nuclear medicine images. The new estimate is based on a nonlinear filtering scheme applied in the wavelet domain. This

Supported by the National Science Foundation, grant no. MIP94-57438, and the Office of Naval Research, grant no. N00014-95-10849. is in marked contrast to classical linear filtering, which has a simple interpretation in the frequency domain. The wavelet domain filter is essentially a spatially-varying filter that automatically adjusts to the local signal and noise structure. The new estimate based on wavelet domain filtering sacrifices the unbiased property of the MLE in order to reduce the variance of the estimate. By allowing a negligible amount of bias, the variance of the estimate can be significantly reduced. Hence, the overall quality of the new estimate is much better than the standard MLE image. Moreover, because the wavelet domain filtering adjusts to the local signal and noise structure, the new estimate is significantly better than linear filtered versions of the MLE - noise is reduced, but important signal structure is not blurred. The paper quantifies this improvement statistically.

The paper is organized as follows. In Section II, we describe the fundamentals of wavelet domain filtering, and describe how it differs from classical linear (space-invariant) filtering methods. In Section III, we provide a novel wavelet-domain filter design method that minimizes two important error measures simultaneously, thus providing an optimal wavelet domain filter. In Section IV, we assess the performance of our new method in several numerical simulations. We also demonstrate the performance of the new method on clinical nuclear medicine data. Conclusions and future avenues of research are discussed in Section $\mathrm{V}$.

\section{Wavelet Domain Filtering}

\section{A. The Wavelet Transform}

The one-dimensional wavelet transform is described as follows [2]. The wavelet decomposition uses basis functions that are translates and dilates of a prototype wavelet. This provides a natural tiling of the time-frequency plane where high frequencies are analyzed with short time-duration basis functions and low frequencies with long time-duration basis functions. A prototype wavelet function and translates and dilates of this prototype are shown in Fig. 1. One of the key features of the wavelet transform is that it provides information about how the signal's frequency content varies over time. The two dimensional wavelet transform is the natural extension of these ideas to spatial-spatial frequency analysis for images.

\section{B. Wavelet-Based Image Filtering}

Filtering in the wavelet domain allows one to perform signal processing operations that are localized in both space and frequency. This can be advantageous in many non-stationary problems, such as radar imaging [3]. Furthermore, empirical evidence shows that wavelet bases generally provide more 
(a)

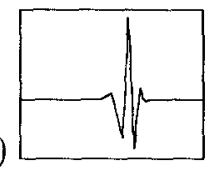

(b)

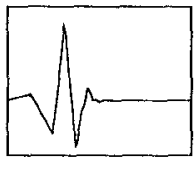

(c)

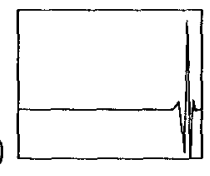

Fig. 1. Wavelet basis functions. (a) Prototype wavelet function. (b) Translated and dilated wavelet for low frequency analysis of signal early in time. (c) Translated and compressed wavelet function for high frequency analysis later in time. Many different prototype wavelet functions can be used in the analysis. Pictured here is the Daubechies-6 wavelet [2].

efficient representations of real-world data than pixel or frequency domain representations. Because wavelets are able to concisely represent complicated signal structure, filtering techniques based on the wavelet decomposition are much better at separating signals from noise than classical approaches based in the pixel or frequency domain. From a theoretical perspective, it has been shown that, because wavelet bases are unconditional bases for wide classes of signals, the wavelet transform is optimal for noise reduction [1]. Wavelet bases are unconditional for many important and practical signal spaces including Besov spaces. Roughly speaking, Besov spaces include signals that are generally smooth except for some possible points of discontinuity. The Besov norm measures signal "smoothness" quite differently from conventional frequency domain notions of smoothness, by allowing local signal discontinuities. Besov spaces are therefore very appropriate for dealing with real-world signals like images.

\section{Wavelet Shrinkage}

The most popular form of wavelet-based filtering is commonly known as Wavelet Shrinkage [1]. The basic wavelet shrinkage program is described as follows. We observe samples $\left\{y_{i}\right\}$ of an unknown function $f$ with additive i.i.d. Gaussian noises $\left\{\eta_{i}\right\}$ :

$$
y_{i}=f(i)+\eta_{i}, \quad i=1, \ldots, n \text {. }
$$

The observations are arranged in a vector $\mathbf{y}=\left[y_{1} \cdots y_{n}\right]^{T}$, and we compute the discrete wavelet transform $\boldsymbol{\theta}=\mathbf{W} \mathbf{y}$. Next, we apply a "soft-threshold" nonlinearity to the wavelet coefficients in $\boldsymbol{\theta}$. The soft-threshold sets very small coefficients to zero and reduces all other coefficients by a fixed amount proportional to the known (or estimated) noise variance. The inverse discrete wavelet transform is applied to the thresholded coefficients to produce a "denoised" signal. An example of Wavelet Shrinkage denoising is shown in Fig. 2.

The unconditional nature of the wavelet basis is crucial to wavelet domain filtering, because it guarantees that filtering always tends to smooth a signal, in the sense of the Besov norm. Smoothing in this sense removes noise while still preserving important image features like edges (see Fig. 2 (c)). Classical linear filtering in the frequency domain removes noise at the expense of blurring important details in the signal (see Fig. 2 (d)). The ability of the wavelet transform to represent signal features that are localized in both space and frequency enables adaptive smoothing that adjusts to the local image structure. (a)

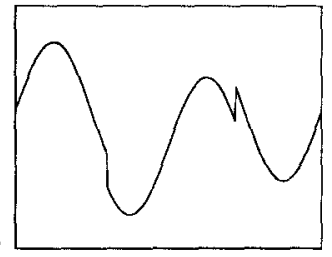

(c)

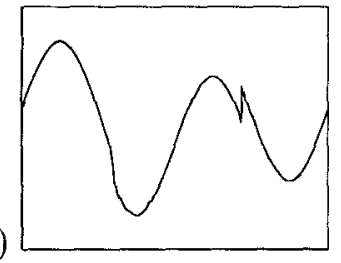

(b)

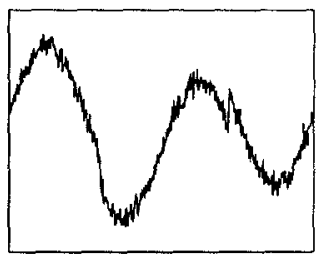

(d)

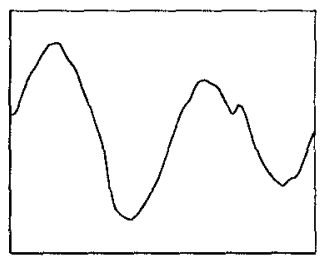

Fig. 2. Wavelet Shrinkage of noisy one-dimensional "HeaviSine" signal [1]. (a) Noise-free signal. (b) Signal plus white Gaussian noise. (c) Denoised signal obtained using nonlinear wavelet shrinkage. (d) For comparison, linear filtering with a Butterworth lowpass filter. Note that nonlinear wavelet soft-thresholding both removes noise and preserves the sharp edge in the signal. Classical linear filtering removes noise but smears the edge.

Wavelet Shrinkage is an optimal filtering scheme for stationary Gaussian white noise environments [1]. Unfortunately, in many problems of interest, including nuclear medicine imaging, the noise is non-Gaussian and non-stationary. In such instances, we must employ more sophisticated filtering methods. The next section describes a novel method for designing optimal wavelet domain filters.

\section{Optimal Wavelet Domain Filter Design Via Cross-Validation}

\section{A. Wavelet Domain Filtering}

Wavelet domain filtering is achieved by the following procedure. First, we compute the two-dimensional wavelet transform of the nuclear medicine image. If $f$ is the original image, the wavelet transform is denoted by

$$
\mathbf{w}=\mathbf{W f} .
$$

The wavelet transformed image $w$ has the same dimensions as $f$. We denote the $i, j$-th pixel in $f$ by $f_{i, j}$. Similarly, the $i, j$-th "wavelet coefficient" is denoted by $\mathbf{w}_{i, j}$. The coefficient $\mathbf{w}_{i, j}$ is a real-valued quantity that reflects the contribution of $i, j$-th wavelet basis function that is present in the image $f$.

With $\mathbf{w}$ in hand, we can filter (or attenuate) the contributions of particular wavelet basis functions by multiplying the corresponding coefficient by a number $0 \leq \mathbf{h}_{i, \jmath} \leq 1$. That is, we modify $\mathrm{w}_{i, j}$ according to

$$
\widetilde{\mathbf{w}}_{i, j}=\mathbf{h}_{i, j} \mathbf{w}_{i, j} .
$$

Setting $\mathbf{h}_{i, j}=0$ completely removes the contribution of this wavelet basis function; setting $\mathbf{h}_{i, j}=1$ leaves it unaltered. Choosing $0<\mathbf{h}_{i, j}<1$ attenuates the contribution of the $i, j$-th wavelet basis accordingly. 
After modifying the wavelet coefficients in this manner, we compute the inverse wavelet transform, denoted by

$$
\tilde{\mathbf{f}}=\mathbf{W}^{-1} \tilde{\mathbf{w}} \text {. }
$$

The new image $\widetilde{f}$ is called a wavelet domain filtered version of $\mathbf{f}$.

The crucial issue in wavelet domain filtering is how to design the filter. The filter design problem is the specification of the $\mathbf{h}_{i, j}$. In the Wavelet Shrinkage program described in the previons section, the $\mathbf{h}_{i, j}$ are determined by the wavelet coefficients themselves. The Wavelet Shrinkage filter is given by:

$$
\mathbf{h}_{i, j}= \begin{cases}1-\frac{\tau \operatorname{sgn}\left(\mathbf{w}_{i, j}\right)}{\mathbf{w}_{i, j}}, & \text { if }\left|\mathbf{w}_{i, j}\right| \geq \tau \\ 0, & \text { if }\left|\mathbf{w}_{i, j}\right|<\tau,\end{cases}
$$

where $\tau$ is a user-specified threshold (usually related to the known or estimated global noise variance). The important thing to note about Wavelet Shrinkage is that this filter, in effect, performs an identical nonlinear transformation on each wavelet coefficient. If the noise in the image is white, then the noise power is the same in each wavelet coefficient, and this procedure is asymptotically minimax optimal [1]. If the noise is not white, then the noise power will differ between wavelet coefficients. Wavelet Shrinkage does not adjust to these differences.

The noise in nuclear medicine is not white. Considering only the noise due to low counts, the noise is larger in pixels with higher intensity. This spatial variation in noise power must be accounted for in the wavelet domain filter design. Given the signal and noise powers, a natural choice for a wavelet domain filter is

$$
\mathbf{h}_{i, j} \approx \begin{cases}1, & \text { if SNR in } \mathbf{w}_{i, j} \text { is high } \\ 0, & \text { if SNR in } \mathbf{w}_{i, j} \text { is low. }\end{cases}
$$

Next, we describe a design procedure that results in an optimal filter of this form.

\section{B. Optimal Filter Design via Cross-Validation}

Cross-validation is a standard procedure for assessing the performance of an estimator [4]. The idea behind cross-validation is to compute an estimate using only part of the data, then validate the estimate by assessing how well it predicts the remaining data. We now formulate a cross-validation procedure for the wavelet domain filter design problem.

Rather than working with the total count image, we subdivide the total acquisition period, $T$ seconds, into $N$ subintervals of $T / N$ seconds each. We then acquire $N$ low counts images in each subinterval, denoted by $\mathbf{f}(1), \ldots, \mathbf{f}(N)$. Commercial imaging machines can easily acquire these images using a "dynamic acquisition" mode. The dynamic acquisition mode is normally reserved for time varying studies. Here, we use the dynamic acquisition to obtain $N$ low count images of a static study. The low-count images are independent and identically distributed in this case. The total count image is related to the low-count images by

$$
\mathbf{f}=\sum_{j=1}^{N} \mathbf{f}(j) .
$$

We use the method of cross-validation and the $N$ low-count images to design an optimal wavelet domain filter as follows. First, compute an image using all but the $k$-th low-count images:

$$
\mathbf{f}^{(k)}=\sum_{j \neq k} \mathbf{f}(j)
$$

The image $\mathbf{f}^{(k)}$ is called a "leaving-one-out" image. Next, we compute the wavelet domain filtered version of $f^{(k)}$ and denote the result by $\widetilde{\mathbf{f}}^{(k)}$. Note that the image $\widetilde{\mathbf{f}}^{(k)}$ depends on the wavelet domain filter coefficients $\mathbf{h}$. The cross-validation idea is to assess how well $\widetilde{\mathbf{f}}^{(k)}$ predicts the low-count image that we have not used, namely $\mathbf{f}(k)$. We measure the prediction error by

$$
e^{(k)}(\mathbf{h})=\left\|\widetilde{\mathbf{f}}^{(k)}-\mathbf{f}(k)\right\|_{F}^{2},
$$

where $\|\cdot\|_{F}^{2}$ is simply the sum of squared errors, pixel by pixel. Note that we view the error as a function of the wavelet domain filter coefficients $\mathbf{h}$. We repeat this process for $k=1,2, \ldots, N$ and consider the predictive sum of squares (PRESS)

$$
V(\mathbf{h})=\sum_{k=1}^{N} e^{(k)}(\mathbf{h})
$$

The PRESS may be viewed as a small-sample optimality criterion measuring the quality of the estimator and the wavelet domain filter coefficients $\mathbf{h}$. The objective is to choose $\mathbf{h}$ to minimize $V(\mathbf{h})$.

It is shown that the optimal $h$, minimizing the PRESS, is given by [4]

$$
\mathbf{h}_{i, j}^{\text {(PRESS) }}=\left(\frac{(N-1)^{2} \mathbf{w}_{i, j}^{2} / N-(N-1){\widehat{\sigma^{2}}}_{i, j}}{(N-1)^{2} \mathbf{w}_{i, j}^{2} / N+\widehat{\sigma^{2}} i, j}\right)_{+}
$$

where $\mathbf{w}_{i, j}^{2}$ is the square of the $i, j$-th wavelet coefficient of the total count image, $\widehat{\sigma}^{2} i, j$ is an estimate variance in the $i, j$ th wavelet coefficient, and $(\cdot)_{+}$denotes positive part (negative values set to zero). The variance is estimated from the $N$ lowcount images according to:

$$
{\widehat{\sigma^{2}}}_{i, j}=\sum_{k=1}^{N}\left(\mathbf{w}_{i, j}(k)-\mathbf{w}_{i, j} / N\right)^{2},
$$

where $\mathbf{w}_{i, j}(k)$ is the $i, j$-th wavelet coefficient of the low count image $\mathbf{f}(k)$.

We call $\mathbf{h}^{\text {(PRESS) }}$ the PRESS-optimal wavelet domain filter, and the call the resulting filtered image, denoted $\widetilde{\mathbf{f}}^{\text {(PRESS)}}$, the PRESS-optimal estimate.

Several comments are in order. First, in addition to minimizing the PRESS, the wavelet domain filter $\mathbf{h}_{i, j}^{\text {(PRESS) }}$ is asymptotically optimal in the mean-square error (MSE) sense. This result follows from a straightforward application of the Strong Law of Large Numbers [4]. This implies that as the total counts increase, $\mathbf{h}_{i, j}^{\text {(PRESS) }}$ tends to the wavelet domain filter that minimizes the MSE, a wavelet domain analog of the classical Wiener filter. Secondly, inspection of (3.10) shows that $h_{i, j}^{\text {(PRESS) }}$ is zero when the estimated SNR

$$
\widehat{S N R}=\frac{(N-1) \mathbf{w}_{i, j}}{N \widehat{\sigma^{2}} i, j}
$$

is less than $0 \mathrm{~dB}$. Furthermore, $\mathbf{h}_{i, j}^{\text {(PRESS) }}$ tends to unity as the estimate SNR increases. Thirdly, the complexity of the optimal filter design is roughly $N$ times that of Wavelet Shrinkage, because we need to compute the wavelet transform of each lowcount image. Finally, note that we require $N>1$ low-count images to compute the optimal wavelet domain filter. However, 
it is possible to design a filter using only the total count image. Due to space limitations, we only briefly sketch the idea here. The details are given in the full-length version of the paper [5]. If we assume that the noise is due to low-count levels alone, then the total count image is an unbiased estimate of both the signal (underlying intensity) and the noise power (variance of the underlying intensity). This is based on the Poisson model of the counts. Hence, we can compute an unbiased estimate of noise power in each wavelet coefficient from the total count image alone. A wavelet domain filter, similar in form to (3.10), can be computed using these estimated noise powers and the wavelet transform of the total count image.

\section{Simulations and Clinical Study}

In this section, we present several simulated imaging experiments that quantify the performance of the cross validation estimation method. In all experiments, the PRESS-optimal wavelet domain filter is designed using the Daubechies-6 [2] prototype wavelet function. This wavelet function is chosen because of its good localization and smoothness properties. The PRESSoptimal filter design is based on splitting the observation period $T$ into $N$ disjoint time intervals to obtain $N$ independent observations of the underlying process. In this application, we can choose $N$ as we like, within the limits of the acquisition system. Our experiments have shown that best results are obtained by choosing $N$ as large as possible. However, increasing $N$ increases the amount of data that must be stored and processed. We have found very little improvement is obtained by choosing $N>10$, and therefore we have selected $N=10$ in all simulations. For more details, please see [5].

\section{A. Experiment 1: Bar Phantom}

To compare the performance of the PRESS-optimal estimate to that of the standard MLE, a simulated $256 \times 256$ resolution bar phantom was designed. The design is similar to the 4-quadrant bar phantom commonly used in nuclear medicine. To provide a meaningful simulation of actual imaging situations, we have smoothed an ideal (sharp edged) bar phantom by a simulated modulation transfer function (for details, see [5]). The smoothed phantom used in this experiment is depicted in Fig. 3.

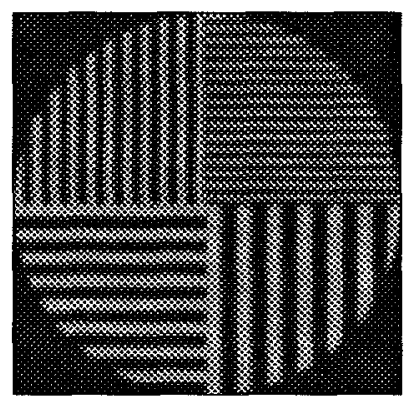

Fig. 3. Smoothed bar phantom used to compare the performance of PRESS-optimal estimates to that of the standard total count $M L E$ image.

We generated Poisson counts according to the smoothed bar phantom, and computed both the MLE and PRESS-optimal estimate of the phantom. We repeated this experiment at different count levels, and generated 50 independent trials at each count level. Using these trials, we computed the mean square error (MSE) of both estimators at each count level. Fig. 4 is a plot of the MSE of both the MLE and PRESS-optimal estimator, as a function of the total counts. Note that in each case the MSE of the PRESS-optimal estimate is less than half that of the MLE. This means that for a given MSE level, the PRESSoptimal estimate requires less than half the counts required for the same error using the MLE. Hence, using the PRESS-optimal wavelet domain filter we may be able to reduce the acquisition time or radioactive dosage level by more than a factor of two and still retain the same image quality.

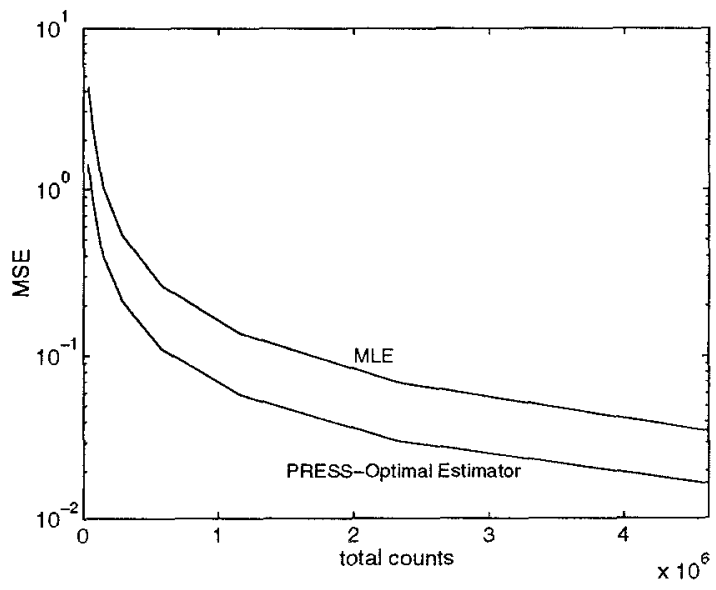

Fig. 4. Comparison of squared error of MLE and PRESSoptimal estimate vs. total counts. MSE of the PRESSoptimal estimator is less than half the MSE of the MLE, at all count levels.

\section{B. Experiment 2: Line Phantom}

To evaluate the potential trade-off between noise reduction and signal smoothing, we simulate a $128 \times 128$ resolution line phantom with lines of varying intensity levels and widths as shown in Fig. 5 (a). The average background level is 8 counts, the maximum line count is 16 (at top), and the minimum line count is 0 (at bottom). Generating counts from this phantom, we compute the MLE and the PRESS-optimal estimate. We compare these to estimates to conventional linear filter smoothing of the MLE in Fig. 5 and Fig. 6 (for details, see [5]).

\section{Clinical Data}

The MLE and PRESS-optimal estimate of a $256 \times 256$ resolution nuclear medicine spinal study are shown in Fig. 7, below. The data for this example were collected using a "dynamic acquisition." Such acquisitions are usually used to collect multiple images for time-varying nuclear medicine studies. For our purposes, the dynamic acquisition was used to collect $N=10$, i.i.d. images of a static nuclear medicine study.

\section{Conclusions and Future Work}

The PRESS-optimal wavelet domain filter is an optimal, data-adaptive filter designed using the method of crossvalidation. The PRESS-optimal filter is also asymptotically 


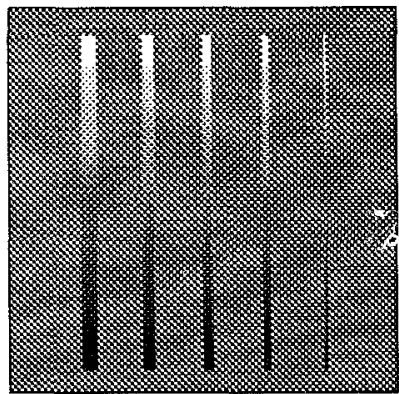

(a)

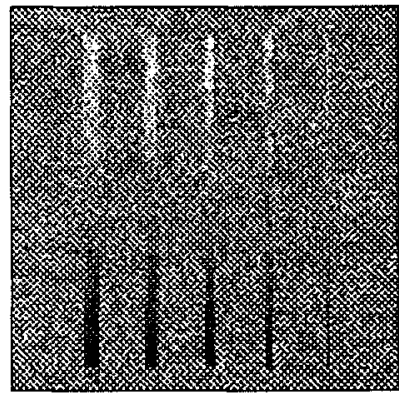

(c)

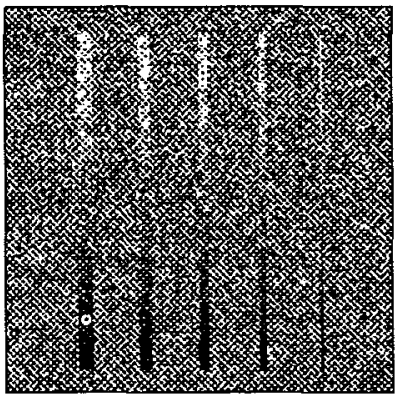

(b)

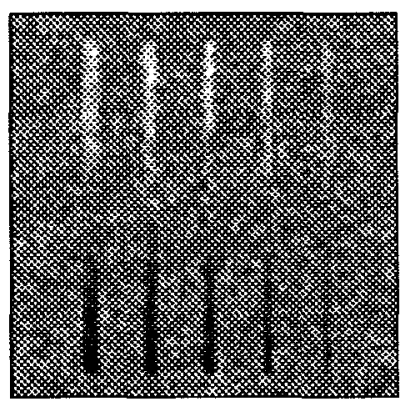

(d)
Fig. 5. Comparison of (a) Phantom, (b) MLE, (c) PRESSoptimal estimate, (d) linear smooth of MLE. Note that the noise in the PRESS-optimal estimate is reduced, but the sharp line structure is preserved. The noise is reduced by linear filtering the $M L E$, but at the expense of reduced contrast in the lines.

optimal in the MSE-sense. We have shown that the PRESSoptimal filter can be applied to nuclear medicine imaging, resulting in much better estimates of the underlying intensity. The PRESS-optimal filter significantly reduces noise in the image, while preserving important structure in the image, contrary to conventional linear filtering. A more comprehensive study and analysis of the PRESS-optimal wavelet domain filter for nuclear medicine is given in [5].

There are several avenues for future work. First, the new filtering method can easily be extended to filtering for tomographic reconstruction. Second, the PRESS-optimal filter can be used in conjunction with "resolution recovery" schemes to reduce the effects of blurring due to collimation, while still reducing noise. Third, the PRESS-optimal wavelet domain filter is applicable to a wide variety of other imaging modalities including CT, PET, and even MRI. We are currently investigating these areas.

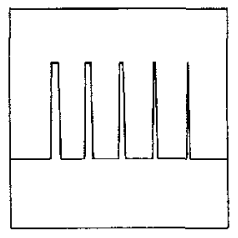

(a)

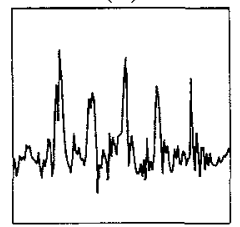

(c)

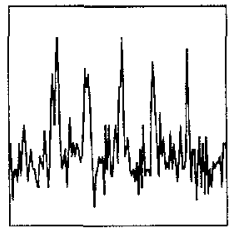

(b)

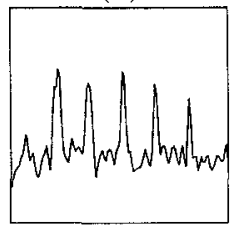

(d)
Fig. 6. Comparison of horizontal profiles from images in Fig. 5. (a) Phantom (b) MLE (c) PRESS-optimal estimate (d) linear smooth of MLE. Noise is reduced in the PRESSoptimal estimate, but the sharp line structure is preserved. Noise is smoothed and reduced by linear filtering the $M L E$ at the expense of smoothing the lines.

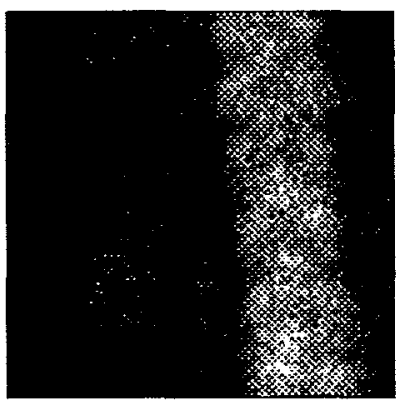

(a)

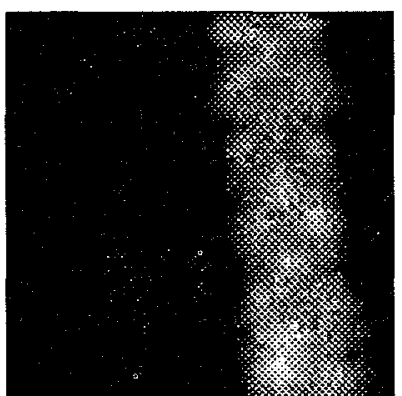

(b)
Fig. 7. Comparison of (a) MLE and (b) PRESS-optimal estimate for spinal study. Notice that the optimal estimate has much better SNR than the MLE and does not oversmooth detail in the spinal structure.

\section{REFERENCES}

[1] D. L. Donoho and I.M. Johnstone, "Adapting to unknown smoothness via wavelet shrinkage," J. American Stat. Assoc., pp. 1200-1224, vol. 90, no. 432, December 1995.

[2] 1. Daubechies, Ten Lectures on Wavelets, CBMS-NSF Series in Applied Mathematics, no. 61, SIAM, 1992.

[3] J. E. Odegard, H. Guo, M. Lang, C. S. Burrus, R. O. Wells Jr., L. M. Novak and M. Hiett, "Wavelet based SAR speckle reduction and image compression," SPIE Symposium on $O E /$ Aerospace Sensing and Dual Use Photonics, pp. 17-21 April 1995, Orlando, FL.

[4] R. D. Nowak, "Optimal signal estimation using crossvalidation," IEEE Signal Processing Letters, vol. 3, no. $12,1996$.

[5] R. D. Nowak, D. J. Nowak, R. G. Baraniuk, and R. S. Hellman, "Wavelet Domain Filtering for Nuclear Medicine Imaging," to be submitted to IEEE Transactions on Medical Imaging, November, 1996. 